\title{
Gastric cancer and adenomatous colorectal polyp concomitant with pyogenic liver abscess and bacteremia
}

\author{
Min Kyu Kang ${ }^{1}$, Hee Jung Kwon ${ }^{2}$, Min Cheol Kim ${ }^{3}$ \\ ${ }^{1}$ Department of Internal Medicine, Yeungnam University College of Medicine, Daegu, Korea \\ ${ }^{2}$ Department of Pathology, Yeungnam University Hospital, Daegu, Korea \\ ${ }^{3}$ Department of Internal Medicine, Yeungnam University Hospital, Daegu, Korea
}

Received: February 27, 2020

Revised: March 24, 2020

Accepted: March 25, 2020

Corresponding author:

Min Kyu Kang

Department of Internal Medicine,

Yeungnam University College of

Medicine, 170 Hyeonchung-ro,

Nam-gu, Daegu 42415, Korea

Tel: +82-53-620-3316

Fax: +82-53-654-8386

E-mail:kmggood111@naver.com

\begin{abstract}
Synchronous gastric cancer and adenomatous colorectal polyp in patients with Klebsiella pneumoniae-induced pyogenic liver abscess (KP-PLA) and bacteremia is a rare presentation. A 58-year-old man with a 6-month history of diabetes mellitus (DM) presented with febrile sensation and dull abdominal pain in the right upper quadrant of the abdomen. Subsequent to laboratory test results and abdominal computed tomography findings, KP-PLA with bacteremia was diagnosed. After intravenous antibiotic administration, his symptoms improved, and upper endoscopy and colonoscopy were performed to evaluate the cause of KP-PLA. Biopsy specimens of the prepyloric anterior wall revealed a moderately differentiated adenocarcinoma. Endoscopic mucosal resection of the colon revealed high-grade dysplasia. Early gastric cancer (EGC) and adenomatous colorectal polyps with high-grade dysplasia concomitant with KP-PLA and bacteremia were diagnosed in our patient who had DM. Intravenous antibiotic treatment for KP-PLA, subtotal gastrectomy for EGC, and colonoscopic mucosal resection for the colon polyp were performed. After 25 days of hospitalization, subtotal gastrectomy with adjacent lymph node dissection was performed. Follow-up ultrasound imaging showed resolution of the abscess 5 weeks post-antibiotic treatment, as well as no tumor metastasis. Upper gastrointestinal endoscopy and colonoscopy should be performed to evaluate gastric cancer in patients with PLA or bacteremia, accompanied with DM or an immunocompromised condition.
\end{abstract}

Keywords: Adenomatous polyps; Klebsiella pneumoniae; Pyogenic liver abscess; Stomach cancer

\section{Introduction}

Pyogenic liver abscess (PLA) is defined as a mass of cystic lesions in the liver with clinical manifestations, including fever, chills, and abdominal pain, which can be caused by either bacterial, fungal, or parasitic organisms [1-3]. The most common causative pathogen of PLA is Klebsiella pneumoniae (KP). KP can invade the liver via many routes, including the biliary tract, hepatic arteries, and portal vein and through direct invasion from an adjacent infected organ [4-6]. Hematogenous PLA is closely associated with colon- ic mucosal injuries such as colon cancer, intestinal tuberculosis, colonic diverticulitis, and inflammatory bowel disease (IBD) $[3,7]$. However, there are few reported cases of PLA with gastric cancer $[3,7]$.

Herein, we present a rare case of early gastric cancer (EGC) and adenomatous colonic polyp with high-grade dysplasia concomitant with KP-induced PLA (KP-PLA) and bacteremia in a patient with diabetes mellitus $(\mathrm{DM})$. 


\section{Case}

This study was approved by the Institutional Review Board of the Yeungnam University Hospital (IRB No: 2019-10-031). All authors declare that written informed consent was obtained from the patient for publication of this case report and accompanying images.

A 59-year-old man with a history of continuous dull pain in the right upper quadrant of the abdomen and chilling sensations for the previous 2 days visited our hospital. He had a 6-month history of DM and 2-year history of hypertension, for which he had not been prescribed any medication. His vital signs were as follows: blood pressure, 142/86 mmHg; heart rate, 98 beats/min; respiratory rate, 22 breaths $/ \mathrm{min}$; and body temperature, $39.6^{\circ} \mathrm{C}$. Physical examination revealed tenderness in the right upper quadrant of the abdomen without a positive Murphy's sign. Blood chemistry results were as follows: white blood cell count, $15,520 / \mu \mathrm{L}$ (neutrophil count $82.8 \%$, lymphocyte count $6.8 \%$ ); hemoglobin, 11.8 $\mathrm{g} / \mathrm{dL}$; platelets, $188 \times 10^{3} / \mu \mathrm{L}$; erythrocyte sedimentation rate, $120 \mathrm{~mm} / \mathrm{hr}$ (range, <25 mm/hr); C-reactive protein, $32.3 \mathrm{mg}$ / $\mathrm{dL}$ (range, <0.5 mg/dL); total bilirubin, $1.16 \mathrm{mg} / \mathrm{dL}$; aspartate aminotransferase, $102 \mathrm{IU} / \mathrm{L}$; alanine aminotransferase, $87 \mathrm{IU} / \mathrm{L}$; alkaline phosphatase, $130 \mathrm{IU} / \mathrm{L}$; gamma-glutamyl transferase, 56 $\mathrm{IU} / \mathrm{L}$; albumin $3.66 \mathrm{~g} / \mathrm{dL}$; and creatinine, $0.7 \mathrm{mg} / \mathrm{dL}$. Abdominal computed tomography revealed a low attenuated lesion measuring approximately $2.1 \times 1.8 \mathrm{~cm}$ in size with an irregular margin in the eight segments of the liver with no intrahepatic biliary abnormalities (Fig. 1). The patient was diagnosed with PLA, and antibiotics, including intravenous ceftriaxone and metronidazole,

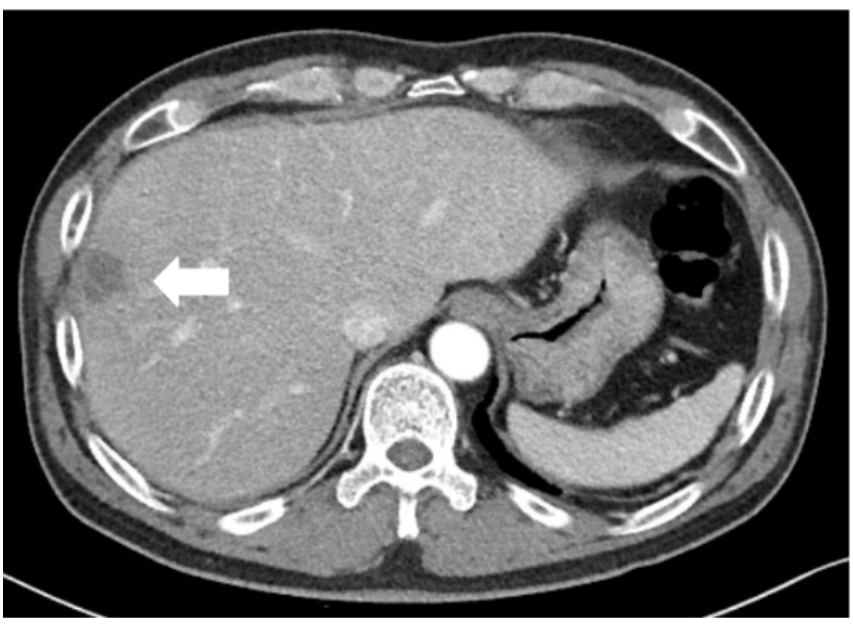

Fig. 1. Initial abdominal computed tomography shows a low attenuated lesion (arrow) measuring approximately $2.1 \times 1.8 \mathrm{~cm}$ in size with an irregular margin in the right liver lobe in the portal phase. were preemptively administered. His clinical symptoms improved, and after KP was identified in blood cultures, ceftriaxone monotherapy was administered.

After 15 days of hospitalization, upper endoscopy and colonoscopy were performed to determine the cause of KP-PLA. Upper endoscopy and colonoscopy revealed ill-defined nodular lesions measuring approximately $2 \mathrm{~cm}$ in size from the anterior wall to the lesser curvature of the prepyloric antrum and a pedunculated polyp in the sigmoid colon measuring approximately $1.5 \mathrm{~cm}$ in size (Fig. 2A, 2B). Biopsy specimens of the prepyloric anterior wall revealed a moderately differentiated adenocarcinoma. Endoscopic mucosal resection of the colon revealed high-grade dysplasia (Fig. 2C, 2D). After 25 days of hospitalization, the patient underwent subtotal gastrectomy with adjacent lymph node dissection. His final diagnosis was a moderately differentiated adenocarcinoma in type 1 EGC (pT1aN0) that was classified by the American Joint Committee on Cancer. Follow-up ultrasound imaging showed resolution of the abscess 5 weeks post-antibiotic treatment and no tumor metastasis.

\section{Discussion}

Liver abscesses, characterized by the spreading of various infec-
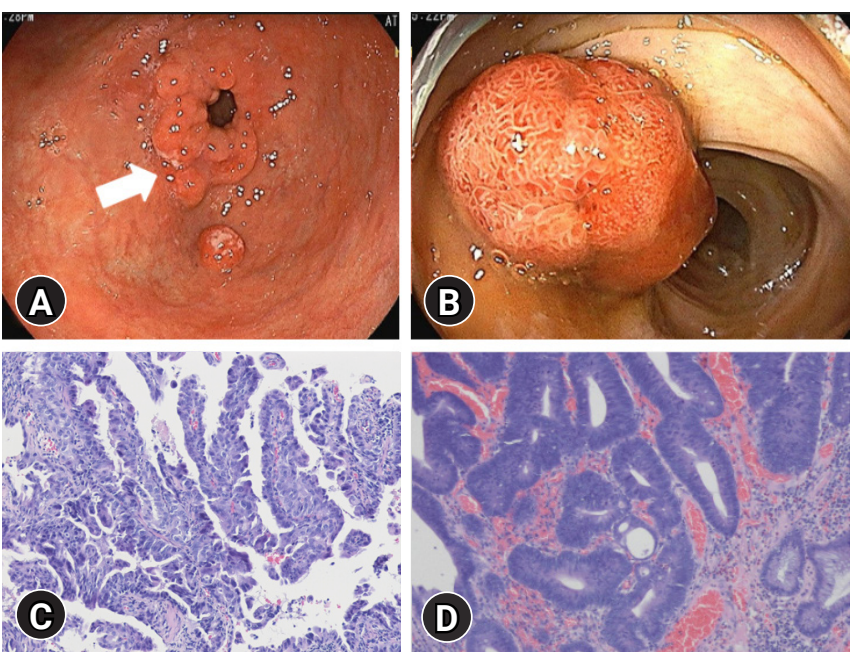

Fig. 2. (A) Upper gastroscopy shows several nodular lesions (arrow) measuring $2 \mathrm{~cm}$ in size with an irregular margin with erosion from the anterior wall to the lesser curvature of the prepyloric antrum. (B) Colonoscopy shows a pedunculated polyp measuring approximately $1.5 \mathrm{~cm}$ in size at the sigmoid colon. (C) The prepyloric nodular lesions show moderately differentiated adenocarcinoma (hematoxylin and eosin [H\&E] stain, $\times 100$ ). (D) The resected colon polyp shows tubular adenoma with highgrade dysplasia. Tumor cells have hyperchromatic nuclei, with cribriform pattern (H\&E stain, $\times 100)$. 
tious pathogens via either the biliary tract, portal vein, or hepatic artery; infection from an adjacent organ; or the accumulation of exudate, are grouped into either PLA or amebic liver abscess $[1,2]$. The recent improvement in public health in Korea has resulted in reducing the prevalence of amebic liver abscess, whereas PLA accounts for $73 \%-96 \%$ of liver abscesses, with KP being the primary causative pathogen [8]. In particular, immunocompromised patients with KP-PLA and conditions such as DM or cancer are at a risk of severe complications, including bacteremia, septic shock, and multiple organ failure [8].

Classically, PLA pathogenesis develops because of an ascending bacterial infection acquired via the biliary tract and typically originates from pylephlebitis accompanied by appendicitis and cholangitis owing to biliary tract surgery or gallbladder stones $[5,6,8]$. Hematogenous PLA is acquired from the hepatic portal vein or artery, which occurs because of a mucosal injury owing to colon cancer, colonic diverticulitis, or IBD $[9,10]$. Through this same mechanism, gastric cancer can lead to PLA via the hematogenous spread of infection from the hepatic portal vein $[3,7]$.

However, because gastric acid eliminates the main causative pathogens, including KP, PLA rarely occurs despite the disruption of mucosal barriers caused by conditions such as gastric ulcers in immunocompetent hosts. Therefore, immunosuppressive conditions, including DM or malignant tumors, may accompany PLA and allow for the migration of pathogens via the compromised gastric mucosa, leading to distant-site infections such as KP-PLA [11].

In our study, mucosal injury owing to EGC enabled the hematogenous spread of KP via the portal vein, leading to KP-PLA with bacteremia. The putative mechanisms underlying KP-PLA with bacteremia are concomitant immunosuppressive conditions such as DM and gastric cancer. As previously mentioned, immunosuppressive conditions may be related to reduced eradication rates of stomach bacteria and reduced opsonized functions of the reticular endothelial systems such as Kupffer cells in the liver [7]. Finally, the decreased phagocytic activity associated with a weakened immune system may have led to the development of PLA with bacteremia in our case.

This study had some limitations. First, adenomatous colorectal polyps with high-grade dysplasia as precancerous lesions are associated with the ascending hematogenous spread of bacterial infection via the portal vein. However, definite evidence of mucosal injury of the colon polyp unlike EGC was not documented in the pathology report. Second, it is unclear whether the major cause of PLA is DM or mucosal injury, and it is difficult to explain the cause-and-effect relationship. It is clear that DM alone is a trigger for PLA. Despite the short duration of DM, mucosal injury in pa- tient with DM is thought to accelerate the formation of PLA.

To the best of our knowledge, this is the first English-written case of EGC and adenomatous colorectal polyp with high-grade dysplasia concomitant with KP-PLA and bacteremia. Based on our case findings, upper gastrointestinal endoscopy can be performed for immunocompromised patients or patients with DM and PLA or bacteremia to screen for gastric cancer. However, further studies are needed to establish the association between PLA and gastric cancer in patients with PLA.

\section{Acknowledgments}

\section{Conflicts of interest}

No potential conflict of interest relevant to this article was reported.

\section{Funding}

This work was supported by the 2019 Yeungnam University Research Grant.

\section{Author contributions}

Conceptualization: MKK, MCK; Data curation: all authors; Formal analysis: MCK; Methodology: MKK, MCK; Investigation: MCK; Project administration, HJK; Visualization: HJK; Writing-original draft, Writing-review \& editing: MKK, MCK.

\section{ORCID}

Min Kyu Kang, https://orcid.org/0000-0002-1435-3312

Hee Jung Kwon, https://orcid.org/0000-0002-8800-7690

Min Cheol Kim, https://orcid.org/0000-0002-2234-8070

\section{References}

1. Li J, Zhao D, Lei L, Zhang L, Yu Y, Chen Q. Liver abscess caused by ingestion of fishbone: a case report. Medicine (Baltimore) 2019;98:e16835.

2. Morioka H, Iguchi M, Kuzuya T, Mikamo H, Yagi T. Recurrent bacteremia and liver abscess caused by Clostridium difficile: a case report. Medicine (Baltimore) 2017;96:e7969.

3. Youn GJ, Choi Y, Kim MJ, Lee JS, Ko UW, Joo YH. Liver abscess and septic complications associated with advanced gastric cancer. Yeungnam Univ J Med 2015;32:38-41.

4. Jung HG, Kim DH, Lee CH. A case of subcapsular liver abscess secondary to perforating ulcer of gastric cancer. Korean J Gastroenterol 2010;56:109-13.

5. Kim SJ, Chu ST, Lee KS, Nam SW, Choi JK, Chung JW, et al. Metastatic endophthalmitis and thyroid abscess complicating 
Klebsiella pneumoniae liver abscess. Clin Mol Hepatol 2018; 24:88-91.

6. Lee KW, Kim HY, Kim CW, Kim YK, Kwon O, Kim MA, et al. Hepatogastric fistula as a rare complication of pyogenic liver abscess. Clin Mol Hepatol 2017;23:87-90.

7. Park DH, Heo NY, Sa-Kong H, Jeong NR, Jeong SJ, Oh SJ, et al. A case of advanced gastric cancer concomitant with pyogenic liver abscess in the patient with subtotal gastrectomy. Korean J Gastroenterol 2017;69:143-6.

8. Jun JB. Klebsiella pneumoniae liver abscess. Infect Chemother
2018;50:210-8.

9. Jeong SW, Jang JY, Lee TH, Kim HG, Hong SW, Park SH, et al. Cryptogenic pyogenic liver abscess as the herald of colon cancer. J Gastroenterol Hepatol 2012;27:248-55.

10. Margalit M, Elinav H, Ilan Y, Shalit M. Liver abscess in inflammatory bowel disease: report of two cases and review of the literature. J Gastroenterol Hepatol 2004;19:1338-42.

11. Lecube A, Pachon G, Petriz J, Hernandez C, Simo R. Phagocytic activity is impaired in type 2 diabetes mellitus and increases after metabolic improvement. PLoS One 2011;6:e23366. 
